\title{
Molecular Imaging of Very Late Antigen-4 in Acute Lung Injury
}

\author{
Joseph Haddad ${ }^{1}$, Joseph D. Latoche ${ }^{2}$, Shubhanchi Nigam ${ }^{1}$, Michael C. Bellavia ${ }^{3}$, Kathryn E. Day ${ }^{2}$, Qin Zhu ${ }^{1}$, \\ W. Barry Edwards ${ }^{1}$, Carolyn J. Anderson ${ }^{1-5}$, and Sina Tavakoli ${ }^{1,2,5}$ \\ ${ }^{I}$ Department of Radiology, University of Pittsburgh, Pittsburgh, Pennsylvania; ${ }^{2}$ Department of Medicine, University of Pittsburgh, \\ Pittsburgh, Pennsylvania; ${ }^{3}$ Department of Bioengineering, University of Pittsburgh, Pittsburgh, Pennsylvania; ${ }^{4}$ Department of \\ Pharmacology and Chemical Biology, University of Pittsburgh, Pittsburgh, Pennsylvania; and ${ }^{5}$ Heart, Lung, Blood, and Vascular \\ Medicine Institute, University of Pittsburgh Medical Center, Pittsburgh, Pennsylvania
}

Inflammation plays a central role in the pathogenesis of acute lung injury (ALI) during both the acute pneumonitis stage and progression into the chronic fibroproliferative phase, leading to pulmonary fibrosis. Currently, there is an unmet clinical and research need for noninvasive ways to monitor lung inflammation through targeting of immunoregulatory pathways contributing to ALI pathogenesis. In this study, we evaluated the role of targeted imaging of very late antigen-4 (VLA-4), as a key integrin mediating the adhesion and recruitment of immune cells to inflamed tissues, in quantifying lung inflammation in a mouse model of lipopolysaccharide-induced ALI. Methods: ALI was induced by a single intratracheal administration of lipopolysaccharide $(10,20$, or $40 \mu \mathrm{g}$ per mouse) in C57BL/6J mice. Control mice were intratracheally instilled with sterile phosphate-buffered saline. VLA-4-targeted PET/CT was performed $24 \mathrm{~h}$ after intravenous injection of a ${ }^{64} \mathrm{Cu}$-labeled high-affinity peptidomimetic ligand referred to as ${ }^{64} \mathrm{Cu}-\mathrm{LLP} 2 \mathrm{~A}$, which is conjugated with the chelator (1,4,8,11-tetraazacyclotetradecane-1-(methane phosphonic acid)-8-(methane carboxylic acid) and a polyethylene glycol 4 linker, at day 2 after the induction of ALI. Ex vivo biodistribution of ${ }^{64} \mathrm{Cu}$-LLP2A was determined by $\mathrm{y}$-counting of harvested organs. The severity of lung inflammation was assessed histologically and by measuring the expression of inflammatory markers in the lung tissue lysates using reverse transcription quantitative polymerase chain reaction. Results: Intratracheal lipopolysaccharide instillation led to an acute inflammatory response in the lungs, characterized by increased expression of multiple inflammatory markers and infiltration of myeloid cells, along with a significant and specific increase in ${ }^{64} \mathrm{Cu}$-LLP2A uptake, predominantly in a peribronchial distribution. There was a strong correlation between the lipopolysaccharide dose and ${ }^{64} \mathrm{Cu}$-LLP2A uptake, as quantified by in vivo PET $(R=0.69, P<0.01)$. Expression levels of both subunits of VLA4 , that is, integrins $\alpha_{4}$ and $\beta_{1}$, significantly correlated with the expression of multiple inflammatory markers, including tumor necrosis factor-a, interleukin-1 $\beta$, and nitric oxide synthase-2, highlighting the potential of VLA- 4 as a surrogate marker of acute lung inflammation. Notably, in vivo ${ }^{64} \mathrm{Cu}$-LLP2A uptake significantly correlated with the expression of multiple inflammatory markers and VLA-4. Conclusion: Our study demonstrates the feasibility of molecular imaging of VLA-4, as a mechanistically relevant target in ALI, and the accuracy of VLA4-targeted PET in quantification of ongoing lung inflammation in a murine model.

Received Jan. 16, 2020; revision accepted Jun. 23, 2020.

For correspondence or reprints contact: Sina Tavakoli, UPMC Presbyterian Hospital, 200 Lothrop St., Suite E200, Pittsburgh, PA 15213.

E-mail: sit23@pitt.edu

Published online Jul. 17, 2020.

COPYRIGHT (C 2021 by the Society of Nuclear Medicine and Molecular Imaging.
Key Words: acute lung injury; very late antigen-4; molecular imaging; inflammation; PET

J Nucl Med 2021; 62:280-286

DOI: 10.2967/jnumed.120.242347

\section{A}

cute lung injury (ALI) is a clinical syndrome of acute hypoxemic respiratory failure associated with diffuse alveolar damage and bilateral radiographic opacities, not attributable to fluid overload or cardiogenic pulmonary edema (1). With an age-adjusted incidence of about 86 per 100,000 person-years, and an in-hospital mortality rate of about $38 \%$ (1), ALI is a major health issue, accounting for approximately 74,500 deaths and 3.6 million hospital days in the United States annually (1).

ALI is a heterogeneous syndrome that may arise from direct exposure to various biologic, chemical, or physical hazards (e.g., pneumonia, aspiration, and toxic fumes) or through indirect insults to the lungs (e.g., sepsis), leading to the disruption of the alveolar-capillary integrity (2). Despite this etiologic heterogeneity, an uncontrolled acute inflammatory response, characterized by excessive accumulation of neutrophils, monocytes, and macrophages, and release of proinflammatory mediators, is a key pathogenic mechanism shared by different etiologies and a major predictor of mortality in ALI $(3,4)$. Additionally, a nonresolving inflammatory response, characterized by continued accumulation of macrophages and fibrocytes or fibroblasts, triggers a fibroproliferative response and is associated with a poor prognosis, such as prolonged ventilator dependence and high mortality (5). This outcome ultimately leads to pulmonary fibrosis, an irreversible sequela of ALI in survivors of the acute pneumonitis phase $(6,7)$.

Molecular imaging is an attractive approach to noninvasively track inflammatory processes contributing to ALI pathogenesis $(8,9)$, through which prognostication and assessment of disease progression or response to therapy may be improved without the need for invasive diagnostic studies, such as bronchoalveolar lavage and lung biopsy. Among the various molecular imaging approaches, detection of the enhanced metabolic activity of activated immune cells, by ${ }^{18} \mathrm{~F}$-FDG PET, has been extensively exploited in inflammatory diseases, including ALI $(10,11)$. However, the limited specificity of ${ }^{18} \mathrm{~F}$-FDG, which targets a nearly ubiquitous metabolic process, has been a challenge to unraveling its biologic and clinical implications in inflammatory diseases (12-14). This 
challenge has triggered recent efforts in imaging specific aspects of immune response in ALI, such as infiltration of neutrophils, monocytes, and macrophages by targeting chemokine receptor- 2 (8), folate receptor- $\beta$ (9), and CD11b (15), with promising results in preclinical studies.

Very late antigen-4 (VLA-4) is a heterodimeric adhesion molecule comprising integrins $\alpha_{4}(\mathrm{CD} 49 \mathrm{~d})$ and $\beta_{1}$ (CD29). Interaction of VLA-4 with its ligands, vascular cell adhesion molecule-1 and fibronectin, plays crucial roles in cell-cell and cell-matrix adhesions required for leukocyte influx in various inflammatory diseases, including pneumonia (16-18) and asthma (19,20). VLA-4 contributes to the recruitment of neutrophils, monocytes, and macrophages in ALI, and its blocking reduces lung inflammation in murine models $(21,22)$. Additionally, strong expression of VLA-4 by immune cells infiltrated into the interstitial and alveolar space has been shown in patients with sepsis-induced ALI (23). Consistently, VLA-4 is crucial for recruitment of neutrophils and lymphocytes to lungs in Streptococcus pneumoniae (16) and Mycobacterium tuberculosis $(17,18)$ pneumonia. These findings strongly support the potential of VLA-4 as a mechanistically relevant and pharmacologically intervenable target for molecular imaging of lung inflammation in ALI.

LLP2A, a peptidomimetic ligand with a high affinity to the activated state of VLA-4 and an excellent safety profile, has been used as the targeting moiety of several preclinical theranostic agents in various oncologic diseases, such as melanoma (24-26) and multiple myeloma $(27,28)$, as well as in imaging immune cells in tuberculosis granulomas in a macaque model (29). Imaging of activated VLA-4 in inflammatory diseases has remained largely unexplored. In this study, we used a previously described LLP2Aderived ${ }^{64} \mathrm{Cu}$-labeled tracer referred to as ${ }^{64} \mathrm{Cu}$-LLP2A, which is conjugated with the chelator (1,4,8,11-tetraazacyclotetradecane-1(methane phosphonic acid)-8-(methane carboxylic acid) and a polyethylene glycol 4 linker (26), to determine whether VLA-4-targeted imaging can quantitatively track the severity of lung inflammation in a murine model of lipopolysaccharide-induced ALI.

\section{MATERIALS AND METHODS}

\section{Mouse Model of ALI}

Adult C57BL/6J mice $(n=29)$ were administered lipopolysaccharide (Escherichia coli, O111:B4) or phosphate-buffered saline (PBS) intratracheally. The studies were performed in accordance with a protocol approved by the University of Pittsburgh Institutional Animal Care and Use Committee.

\section{PET/CT and Quantification of ${ }^{64} \mathrm{Cu}$-LLP2A Uptake}

Tracer synthesis and radiolabeling were previously described (26). Twenty-four hours after instillation of lipopolysaccharide (10, 20, or $40 \mu \mathrm{g}$ ) or PBS ( 2 male mice and 2 female mice in each group), the mice were injected intravenously with ${ }^{64} \mathrm{Cu}-\mathrm{LLP} 2 \mathrm{~A}(6.4 \pm 0.2 \mathrm{MBq})$. Tracer specificity was addressed in separate groups of male mice $(40 \mu \mathrm{g}$ of lipopolysaccharide), imaged with $(n=3)$ or without $(n=4)$ coinjection of approximately $25 \mathrm{nmol}$ of nonlabeled LLP2A. Static PET ( $\sim 10 \mathrm{~min})$ and CT (180 projections, $140-\mathrm{ms}$ exposure, $180^{\circ}$ rotation, $80 \mathrm{kVp}, 500 \mu \mathrm{A}, 78.5 \times 100 \mathrm{~mm}$ field of view) were performed (Inveon; Siemens) $24 \mathrm{~h}$ after ${ }^{64} \mathrm{Cu}$-LLP2A injection. ${ }^{64} \mathrm{Cu}$-LLP2A uptake was quantified as SUV (IRW software). Because of the heterogeneous pattern of lung inflammation, we used $S U V_{\text {max }}$ rather than $S U V_{\text {mean }}$ to avoid underestimation of tracer uptake. To obtain a representation of the total burden of inflammation, the lungs were divided into serial nonoverlapping zones, each comprising 5 slices about $0.8 \mathrm{~mm}$ thick. This yielded 4-5 approximately 4-mm zones after exclusion of apices and bases, which could not be analyzed because of uptake in adjacent organs (thymus and liver). Regions of interest were drawn over the right and left lungs in slices with the highest uptake to determine the $\mathrm{SUV}_{\text {max }}$ in each zone. These $\mathrm{SUV}_{\max }$ results were averaged (4-5 values per each lung) to obtain average $\mathrm{SUV}_{\max }$.

Biodistribution was performed by $\gamma$-counting (Wizard ${ }^{2}$; PerkinElmer) of harvested organs. Data are reported as percentage injected dose per gram of tissue (26).

\section{Gene Expression Assays}

Gene expression was quantified in the right lungs of mice that underwent PET/CT after instillation of 10,20 , or $40 \mu \mathrm{g}$ of lipopolysaccharide or PBS ( 2 male mice and 2 female mice per group), per standard methods (13) using TaqMan primers (Supplemental Table 1; supplemental materials are available at http://jnm.snmjournals.org) and a QuantStudio 3 real-time polymerase chain reaction system (Applied Biosystems). Transcript levels are normalized to 18S ribosomal RNA (Rn18s) (12) and presented relative to the levels in PBS control mice.

\section{Histology}

Immunohistology of the lungs was performed on $10-\mu \mathrm{m}$ cryosections (CM1860; Leica) in 3 male and 3 female mice instilled with $40 \mu \mathrm{g}$ of lipopolysaccharide or PBS, respectively, using commercially available antibodies (Supplemental Table 2). These mice did not undergo imaging, as lung architecture preservation necessitated inflation by fixative, interfering with measurement of lung weights for biodistribution analysis. The slides were photographed using an Axio Vert microscope (Zeiss).

\section{Statistical Analysis}

Statistical analysis was performed using Prism (version 8; GraphPad). Data are presented as mean \pm SEM. D'Agostino-Pearson normality testing revealed a normal distribution of tracer uptake (SUV $\mathrm{Sux}_{\text {max }}$ and biodistribution) but nonnormal distributions of gene expression (except for Cd68 and Itga4). Accordingly, 1-way ANOVA, followed by Fisher exact post hoc testing, was used to compare mean values of tracer uptake in more than 2 groups (i.e., different lipopolysaccharide doses). Nonpaired $t$ testing was performed to compare tracer uptake
FIGURE 1. PET/CT of lung inflammation in lipopolysaccharide-induced ALI. (A) Representative $\mathrm{PET} / \mathrm{CT}$ images of mice $2 \mathrm{~d}$ after intratracheal administration of $40 \mu \mathrm{g}$ of lipopolysaccharide (top row) or PBS (bottom row) demonstrate heterogeneous uptake of ${ }^{64} \mathrm{Cu}$-LLP2A in inflamed lungs, with peribronchial distribution. (B) Quantitative analysis demonstrates significant correlation between ${ }^{64} \mathrm{Cu}$-LLP2A uptake and lipopolysaccharide dose. Data points represent averaged uptake of right and left lungs in each mouse (4 per group; red dot in $20-\mu \mathrm{g}$ lipopolysaccharide group represents technical outlier (female), excluded in all subsequent analysis because of absence of lung inflammation by gene expression assays). LPS = lipopolysaccharide. 


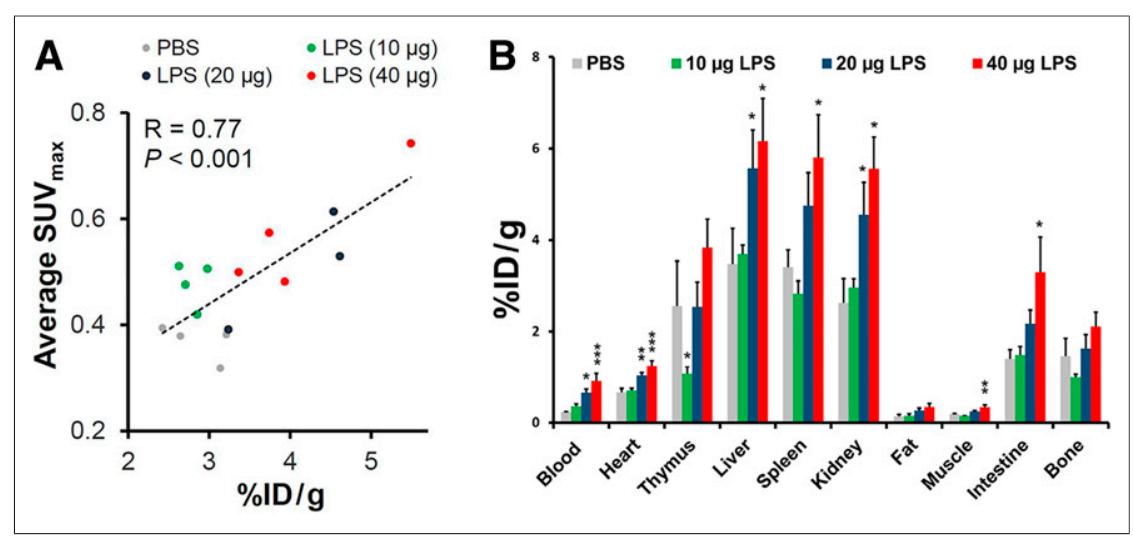

FIGURE 2. Biodistribution of ${ }^{64} \mathrm{Cu}$-LLP2A. (A) In vivo PET-derived quantification of lung ${ }^{64} \mathrm{Cu}$ LLP2A uptake significantly correlates with ex vivo quantification of uptake $2 \mathrm{~d}$ after induction of ALI. Data points represent averaged uptake of right and left lungs in each mouse. (B) Ex vivo biodistribution of ${ }^{64} \mathrm{Cu}$-LLP2A in major organs $24 \mathrm{~h}$ after intravenous administration. Data are expressed as percentage injected dose per gram of tissue (\%ID/g) (3 in 20- $\mu$ g lipopolysaccharide group and 4 in other groups). ${ }^{*} P<0.05$ compared with PBS control group. ${ }^{\star *} P<0.01$ compared with PBS control group. ${ }^{\star \star \star} P<0.001$ compared with PBS control group. LPS $=$ lipopolysaccharide.

between blocked and nonblocked groups. Pearson correlation testing was used to determine the associations between in vivo and ex vivo quantifications of tracer uptake, as well as their associations with lipopolysaccharide doses. Nonparametric Kruskal-Wallis analysis, followed by Benjamini-Yekutieli testing, was used to compare the expression levels of inflammatory markers between the groups. Associations between tracer uptake and markers of inflammation were determined nonparametrically by Spearman testing. $P$ values of less than 0.05 were considered statistically significant.

\section{RESULTS}

\section{Uptake of ${ }^{64} \mathrm{Cu}$-LLP2A Correlates with the Inciting Dose of Lipopolysaccharide-Induced ALI}

$\mathrm{PET} / \mathrm{CT}$ performed $24 \mathrm{~h}$ after injection of ${ }^{64} \mathrm{Cu}-\mathrm{LLP} 2 \mathrm{~A}$ demonstrated heterogeneous tracer accumulation, predominantly localized in a peribronchial distribution, in the mice that were intratracheally instilled with lipopolysaccharide but not the PBS control mice (Fig. 1A). In vivo PET-derived quantification of ${ }^{64} \mathrm{Cu}$-LLP2A uptake (Fig. 1B) demonstrated significant increases in the average $\mathrm{SUV}_{\max }$ in the lungs of mice receiving $40 \mu \mathrm{g}$ of lipopolysaccharide $\left(\mathrm{SUV}_{\max }, 0.57 \pm 0.06 ; P<0.01\right)$ and $20 \mu \mathrm{g}$ of lipopolysaccharide $\left(\mathrm{SUV}_{\max }, 0.51 \pm 0.06 ; P<0.05\right)$, as well as a trend toward significance in those receiving $10 \mu \mathrm{g}$ of lipopolysaccharide $\left(\mathrm{SUV}_{\max }, 0.48 \pm 0.02 ; P=0.08\right)$, versus the PBS control group $\left(\mathrm{SUV}_{\max }, 0.37 \pm 0.02\right)$. Notably, there was a strong correlation between the average $\mathrm{SUV}_{\max }$ and the inciting dose of lipopolysaccharide $(R=0.69, P<0.01)$ (Fig. 1B). No significant difference was present in ${ }^{64} \mathrm{Cu}$-LLP2A uptake between male and female mice (though our study was not powered for sex-specific analysis).

Consistent with in vivo PET-derived quantification, ex vivo quantification by $\gamma$-counting demonstrated an approximately 1.5 fold increase in the ${ }^{64} \mathrm{Cu}$-LLP2A uptake in lungs instilled with $40 \mu \mathrm{g}$ of lipopolysaccharide, versus PBS $(3.63 \pm 0.46$ vs. $2.35 \pm 0.19$ percentage injected dose per gram of tissue, respectively, $P=$ 0.01) (Fig. 2A). To verify whether quantification of in vivo imaging accurately reflects the $\gamma$-counting measure of tracer uptake, as the gold standard, we determined the correlation between average $\mathrm{SUV}_{\max }$ and percentage injected dose per gram of tissue. As shown in Figure 2A, there was a strong correlation between in vivo and ex vivo quantification of ${ }^{64} \mathrm{Cu}$-LLP2A uptake in the lungs $(R=0.77$, $P<0.001)$.

Consistent with a recent report of intratracheal lipopolysaccharide-induced systemic inflammation and enhanced multiorgan uptake of a ${ }^{64} \mathrm{Cu}$-labeled anti-CD11b immunotracer (15), our ex vivo biodistribution studies (Fig. 2B) demonstrated a significant increase in ${ }^{64} \mathrm{Cu}-\mathrm{LLP} 2 \mathrm{~A}$ uptake in several organs, including liver, spleen, and intestines. There was also a significant increase in the blood-pool activity in the mice that received higher doses of lipopolysaccharide (20 and $40 \mu \mathrm{g}$ ), versus the PBS control mice.

Specificity of ${ }^{64} \mathrm{Cu}-\mathrm{LLP} 2 \mathrm{~A}$ uptake was determined by coadministration of an approximately 200 -fold molar excess of nonlabeled LLP2A to mice instilled with $40 \mu \mathrm{g}$ of lipopolysaccharide. This procedure reduced lung uptake of ${ }^{64} \mathrm{Cu}-\mathrm{LLP} 2 \mathrm{~A}$ by about $50 \%$, as determined by $\mathrm{SUV}_{\max }$ and $\gamma$-counting (Supplemental Fig. 1). Interestingly, there were significant decreases in ${ }^{64} \mathrm{Cu}-$ LLP2A uptake in additional organs (e.g., thymus, spleen, and bone), but not in the blood pool, indicating specific uptake of

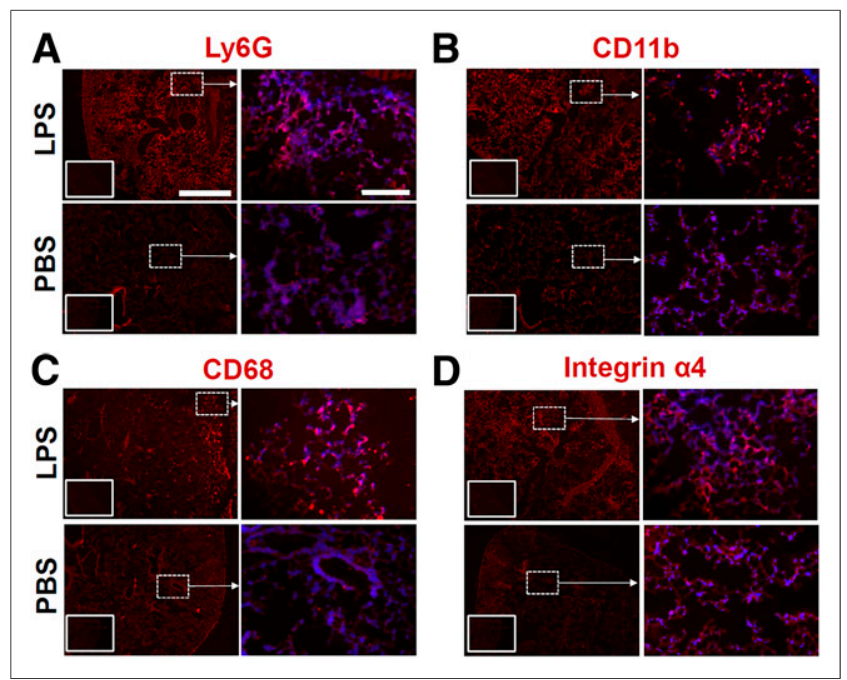

FIGURE 3. Immunohistology in lipopolysaccharide-induced ALI. (A-C) Representative immunofluorescent staining shows intense infiltration of inflamed lungs after lipopolysaccharide-induced ALI (top rows), compared with PBS control lungs (bottom rows), by myeloid cells expressing neutrophilic and monocytic or macrophage markers (in red), including Ly6g (A), CD11b (B), and CD68 (C). (D) Abundance of integrin $a_{4}-$ expressing cells is also increased in ALI. Right columns in each group represent high-magnification images of regions demarcated by dashed boxes in low-magnification images in right columns with overlaid DAPI nuclear staining (blue). Insets in lower right corner of low-magnification images represent negative control staining. Scale bars indicate $1,000 \mu \mathrm{m}$ and $200 \mu \mathrm{m}$ in low- and high-magnification images, respectively. $n=3$ per group. LPS = lipopolysaccharide. 


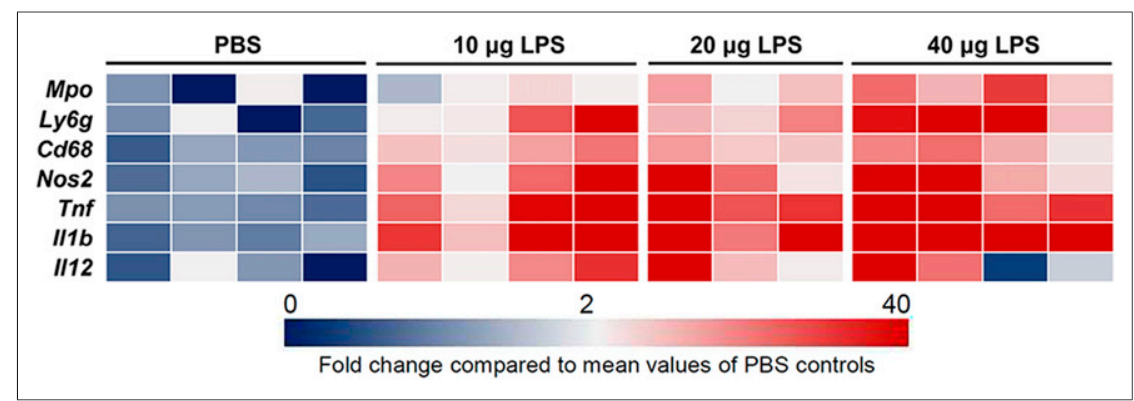

FIGURE 4. Induction of lung inflammation by lipopolysaccharide-induced ALI. Heat map representation confirms marked increase in mRNA transcripts of several inflammatory markers in inflamed lungs $2 \mathrm{~d}$ after induction of ALI. Expression level of each transcript is demonstrated relative to average expression in PBS control group, using color intensity scale ranging from 0 (dark blue) to $>40$ (dark red), with 2 -fold values in white. Analyses were performed for right lung. $n=3$ in 20- $\mu$ g lipopolysaccharide group and 4 in other groups. LPS = lipopolysaccharide.

${ }^{64} \mathrm{Cu}$-LLP2A secondary to lipopolysaccharide-induced systemic inflammation.

\section{VLA-4 Expression Is a Surrogate Marker of Acute} Inflammation in Lipopolysaccharide-Induced ALI

Intense infiltration of immune cells, most notably neutrophils, monocytes, and macrophages, is a critical process in the pathogenesis of ALI (3,4,8,9,21,22). Consistently, our immunofluorescent staining showed an abundance of cells expressing myeloid markers CD11b, Ly6g, and CD68 in the lungs of mice treated with intratracheal

A

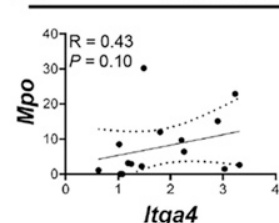

Itga4
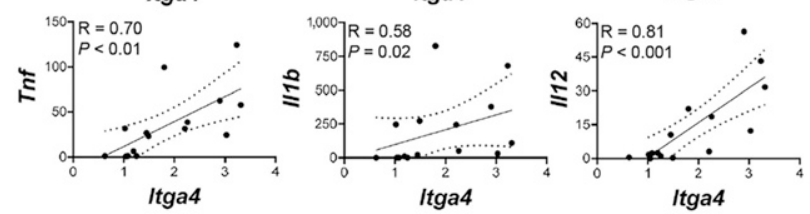

B
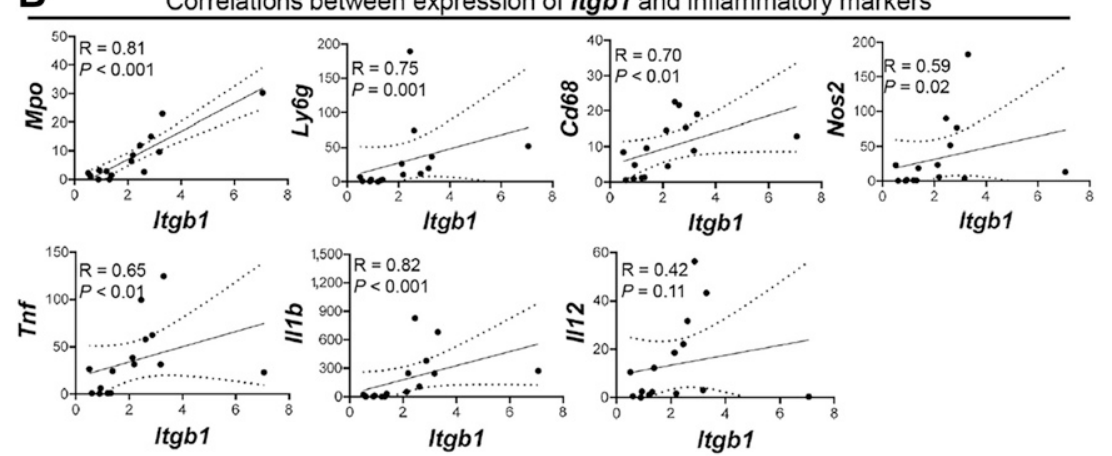

FIGURE 5. Correlations between expression of Itga4 (A) and Itgb1 (B) with markers of lung inflammation. Expression of both $\alpha_{4}$ and $\beta_{1}$ subunits of VLA-4 correlates with expression levels of various markers of neutrophil and monocyte- or macrophage-driven inflammation. Transcript levels are normalized to Rn18s. Analyses were performed for right lung. $n=3$ in $20-\mu g$ lipopolysaccharide group and 4 in other groups. lipopolysaccharide, compared with those receiving PBS (Fig. 3). In addition, there was an increase in the abundance of cells expressing the $\alpha_{4}$ integrin subunit of VLA-4 in lipopolysaccharide-treated versus PBS-treated lungs. We further quantified the severity of lung inflammation by assessing the transcription level of a panel of inflammatory markers. As summarized in a heat map (Fig. 4), there was a robust increase in the expression of several markers of acute inflammation, most pronounced in the 40$\mu \mathrm{g}$ lipopolysaccharide group versus the PBS control group, including Cd68 (15fold), myeloperoxidase (Mpo) (18-fold), $\mathrm{Ly} 6 \mathrm{~g}$ (72-fold), nitric oxide synthase 2 (Nos2) (73-fold), tumor necrosis factor- $\alpha$ (Tnf) (70-fold), interleukin-1 $\beta$ (Illb) (500-fold), and interleukin-12 (Il12) (17-fold).

To determine whether VLA-4 expression quantitatively tracks the severity of lung inflammation, thereby serving as a surrogate target for imaging, we assessed the correlations between VLA-4 subunits (i.e., integrin $\alpha_{4}$ [Itga4] and integrin $\beta_{1}$ [Itgbl]) and the above markers of inflammation (Figs. 5A and 5B). Itga4 mRNA expression was significantly correlated with levels of $\operatorname{Ly6g}(R=0.63, P=0.01), C d 68(R=$ $0.85, P<0.001), \operatorname{Nos} 2(R=0.80, P<0.001), \operatorname{Tnf}(R=0.70, P<$ $0.01)$, $I l 1 b(R=0.58, P=0.02)$, and $I l 12(R=0.81, P<0.001)$. There was also a trend toward modest correlations between the expression of Itga4 and Mpo $(R=0.43, P=0.10)$. Moreover, Itgbl expression was significantly correlated with $M p o(R=0.81, P<0.001)$, $\operatorname{Ly6g}(R=0.75, P=0.001), C d 68(R=0.70$, $P<0.01), \operatorname{Nos} 2(R=0.59, P=0.02), T n f$ $(R=0.65, P<0.01)$, and $I l 1 b(R=0.82$, $P<0.001)$.

\section{In Vivo Uptake of ${ }^{64} \mathrm{Cu}$-LLP2A Parallels the Severity of Acute Inflammation in ALI}

We next determined whether in vivo uptake of ${ }^{64} \mathrm{Cu}-\mathrm{LLP} 2 \mathrm{~A}$ reflects the extent of ongoing lung inflammation by assessing correlations between $\mathrm{SUV}_{\max }$ and the expression of VLA4 and other inflammatory markers in the corresponding right lung (Fig. 6). Significant correlations were noted between ${ }^{64} \mathrm{Cu}$ LLP2A uptake and expression of Mpo $(R=$ 0.68, $P<0.01), C d 68(R=0.53, P=$ $0.04), \operatorname{Nos} 2(R=0.63, P=0.01)$, and $I l 1 b$ $(R=0.60, P=0.01)$. Correlations between $\mathrm{SUV}_{\max }$ and other markers also trended toward significance, including Itga4 $(R=$ $0.51, P=0.05)$, Igtbl $(R=0.46, P=$ $0.08), \operatorname{Tnf}(R=0.49, P=0.06)$, and $I l 12$ ( $R=0.44, P=0.10$ ), though these were modest in strength.

\section{DISCUSSION}

Our study demonstrated significant correlations between the expression of VLA-4 and multiple inflammatory markers in a mouse 


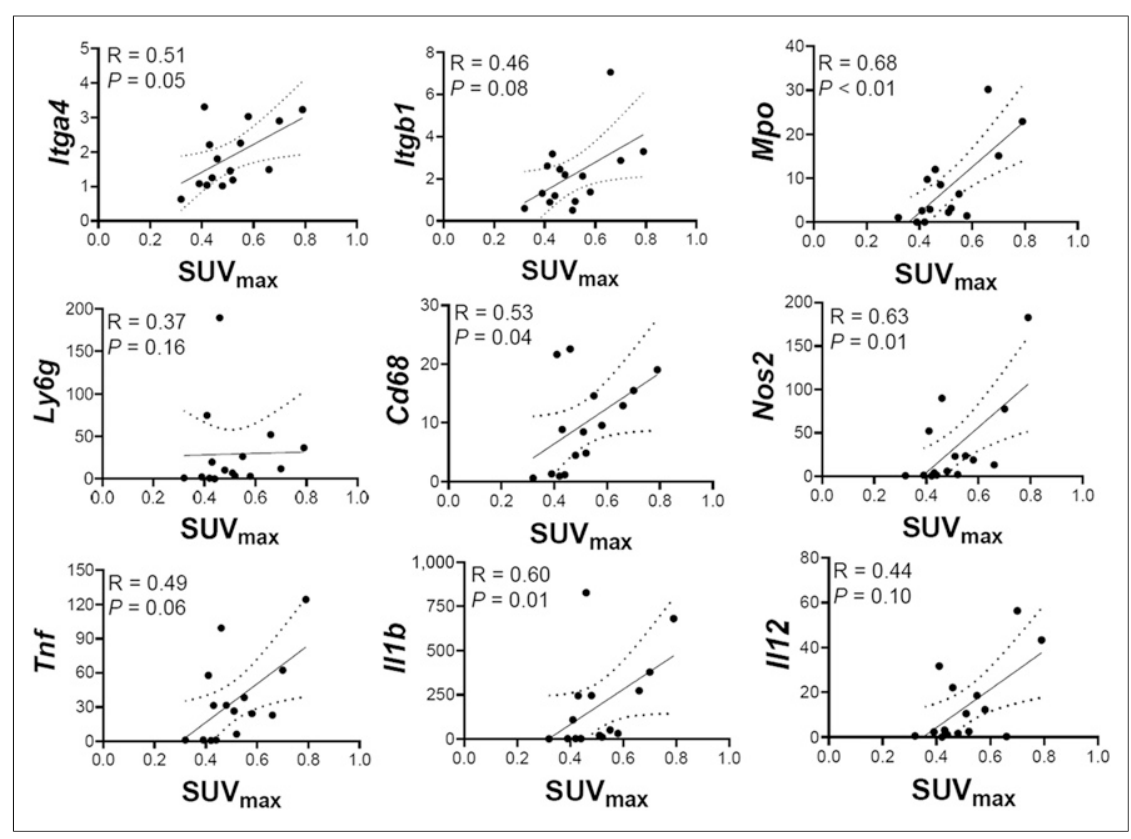

FIGURE 6. Correlations between ${ }^{64} \mathrm{Cu}-\mathrm{LLP} 2 \mathrm{~A}$ uptake and markers of lung inflammation. In vivo quantification of ${ }^{64} \mathrm{Cu}$-LLP2A uptake demonstrates significant correlations between average SUV $_{\max }$ and expression of Mpo, Cd68, Nos2, and $I / 1 \mathrm{~b}$ in right lungs. There are also trends toward significance between average lung SUV $\max$ and expression of $\operatorname{Tnf}(P=0.06)$ and $/ / 12(P=0.10)$. Transcript levels are normalized to Rn18s. $n=3$ in $20-\mu \mathrm{g}$ lipopolysaccharide group and 4 in other groups.

model of lipopolysaccharide-induced ALI, highlighting its role as a biomarker of acute lung inflammation. We also showed the feasibility, specificity, and accuracy of quantitative in vivo VLA-4-targeted PET, using ${ }^{64} \mathrm{Cu}$-LLP2A, to track the severity of inflammation in ALI throughout a range of inciting lipopolysaccharide doses.

An acute inflammatory response, manifesting as intense infiltration of lungs by immune cells (predominantly neutrophils and monocytes or macrophages (3)) and release of proinflammatory mediators, is a hallmark of ALI $(3,30)$. The release of various proteolytic enzymes, reactive oxygen and nitrogen species, and cytokines by infiltrated leukocytes plays a critical role in ALI pathogenesis through disrupting the alveolar endothelial-epithelial barrier. Additionally, a longstanding nonresolving inflammatory response causes irreversible lung damage that ultimately leads to lung fibrosis (6). Consistent with previous reports $(8,9,21,24,26)$, immunohistology demonstrates accumulation of myeloid cells in ALI. This finding was confirmed quantitatively by distinctly higher transcript levels of myeloid markers in lipopolysaccharide-treated lungs than in PBS controls. Moreover, our data indicated a robust increase in the expression of several other markers of acute inflammation in ALI, including Mpo, Tnf, Illb, Ill2, and Nos2.

Increasing preclinical evidence supports the promising role of molecular imaging in noninvasive detection of various aspects of myeloid cell biology in ALI $(8,9,15)$. For example, ${ }^{64} \mathrm{Cu}$-labeled anti-CD11b (15) and ${ }^{64} \mathrm{Cu}-D O T A-E C L 1 i, a{ }^{64} \mathrm{Cu}$-labeled tracer targeting chemokine receptor-2 (8), have been successfully used in imaging of lipopolysaccharide-induced ALI. Expression of folate receptor- $\beta$ by activated macrophages has also been exploited for optical imaging of lipopolysaccharide-induced lung inflammation (9).

Here, we evaluated the role of targeted imaging of VLA-4 as a key adhesion molecule in promoting the migration of immune cells to inflammatory sites to quantify acute lung inflammation.
The number of VLA-4-expressing hematopoietic progenitor cells increases and peaks by approximately $48 \mathrm{~h}$ after intratracheal administration of lipopolysaccharide, coinciding with the raised level of cytokines in bronchoalveolar lavage fluid (31). VLA-4-expressing neutrophils and monocytes have also been shown to infiltrate lung interstitium and alveolar space during ALI $(22,23,32)$. The mechanistic role of VLA-4 in recruitment of myeloid cells to the lungs has been confirmed through pretreatment with an anti-VLA-4 blocking antibody in a murine model of lipopolysaccharide-induced ALI (22,32). Consistently, our results demonstrated significant correlations between the expression levels of $\alpha_{4}$ and $\beta_{1}$ integrin subunits of VLA-4 and multiple markers of acute inflammation, supporting the premise to noninvasively target VLA-4 as a mechanistically relevant imaging biomarker of lung inflammation. Correlations of $\alpha_{4}$ and $\beta_{1}$ subunits with a few inflammatory markers were modest but trended toward significance, presumably because of differential expression of VLA-4 subunits among different subtypes or activation states of leukocytes.

Several pharmacokinetic and pharmacodynamic features of LLP2A have made it an excellent theranostic agent, including its high and selective affinity toward activated VLA-4 (in the low nanomolar range, promoting receptor-mediated tracer internalization), its resistance to plasma proteases conferred by its unnatural amino acids, and its compatibility with various derivatization, allowing achievement of high molar activity, optimal bioavailability, and excellent safety profile $(27,33)$. Although LLP2A-derived theranostic agents have been extensively investigated in oncologic studies (24-28), their roles in molecular imaging of inflammatory diseases has remained largely unexplored (29). Considering the well-established role of VLA-4 in the recruitment of immune cells, specifically its role in leukocyte influx into inflamed lungs $(16-23,34)$, we sought to determine whether ${ }^{64} \mathrm{Cu}-\mathrm{LLP} 2 \mathrm{~A}$ PET allows for quantitative imaging of inflammation in ALI.

In this study, we showed that focal areas of ${ }^{64} \mathrm{Cu}$-LLP2A uptake localize predominantly in a peribronchial distribution, corresponding to the expected pattern of inflammation in intratracheal lipopolysaccharide-induced ALI (35). Given this spatial heterogeneity in the inflammatory response, we quantified ${ }^{64} \mathrm{Cu}$-LLP2A uptake as the average of $\mathrm{SUV}_{\max }$ in nonoverlapping zones of each lung, as an indicator of overall burden of lung inflammation. This quantification approach avoids underestimation of the inflammatory burden using $\mathrm{SUV}_{\text {mean }}$, which is influenced by inclusion of noninflamed lung regions. The significant correlations between in vivo quantification of ${ }^{64} \mathrm{Cu}$-LLP2A and ex vivo uptake determined by $\gamma$-counting, as well as the administered lipopolysaccharide dose, support the accuracy of this quantification approach to track the biodistribution and extent of lung inflammation. A notable finding is the presence of significant correlations between in vivo quantification of ${ }^{64} \mathrm{Cu}-\mathrm{LLP} 2 \mathrm{~A}$ and expression levels of integrins $\alpha_{4}$ and $\beta_{1}$, as well as multiple proinflammatory markers, supporting the potential role of ${ }^{64} \mathrm{Cu}$-LLP2A in imaging acute inflammation in ALI. The strengths of correlations between ${ }^{64} \mathrm{Cu}-\mathrm{LLP} 2 \mathrm{~A}$ uptake 
and a few inflammatory markers, including Mpo, Nos2, and $I l 1 b$, were stronger than others, particularly Ly6 $g$ and $I l 12$. This finding suggests that VLA-4 expression or activation may be more strongly associated with specific subsets or activation states of immune cells in inflamed lungs. Considering the phenotypic diversity of recruited immune cells in ALI and the complex regulation of inflammatory markers beyond their transcription, further investigations are required to address this possibility.

Consistent with the results of a recent study involving systemic inflammation induced by intratracheal lipopolysaccharide detectable by ${ }^{64} \mathrm{Cu}$-labeled anti-CD11b PET (15), our biodistribution analysis showed higher levels of ${ }^{64} \mathrm{Cu}-\mathrm{LLP} 2 \mathrm{~A}$ in multiple organs, including liver, spleen, intestine, kidneys, and heart, as well as blood, indicating a multiorgan inflammatory response to lipopolysaccharide. Similarly, blood-pool activity was higher in mice receiving high doses of lipopolysaccharide (20 and $40 \mu \mathrm{g}$ ), presumably secondary to peripheral blood leukocytosis, which occurs as part of the systemic inflammatory response during the acute phase of lipopolysaccharideinduced ALI (35), and increased binding of tracer to VLA4-expressing circulating cells. We also noted a significant decrease in thymic uptake of ${ }^{64} \mathrm{Cu}-\mathrm{LLP} 2 \mathrm{~A}$ in response to the low dose $(10 \mu \mathrm{g})$ of lipopolysaccharide, speculatively representing the mobilization of VLA-4-expressing cells, including lymphocytes.

\section{CONCLUSION}

Consistent with the mechanistic role of VLA-4 in leukocyte recruitment to inflamed lungs (16-22,34), our study highlights the role of VLA-4 as a biomarker of acute lung inflammation and the feasibility of quantitative ${ }^{64} \mathrm{Cu}-\mathrm{LLP} 2 \mathrm{~A}$ PET in noninvasive tracking of ongoing inflammation in ALI. The excellent safety profile and correlation of ${ }^{64} \mathrm{Cu}$-LLP2A uptake in lungs with lipopolysaccharide dose underscores the translational potential of VLA4-targeted imaging, which may be used toward a precision approach for risk stratification and management of ALI.

\section{DISCLOSURE}

This work was supported by NIH-NHLBI K08-HL144911 and a Seed Fund grant from the University of Pittsburgh to Sina Tavakoli. This work was also supported by NIH-NCI Cancer Center support grant P30-CA047904 (In Vivo Imaging Facility) and by NIH-NIAID R01-AI118195. No other potential conflict of interest relevant to this article was reported.

\section{KEY POINTS}

QUESTION: Can VLA-4-targeted PET noninvasively monitor the severity of lung inflammation in ALI?

PERTINENT FINDINGS: Lung uptake of ${ }^{64} \mathrm{Cu}$-LLP2A correlated with the inciting dose of intratracheal lipopolysaccharide and expression of proinflammatory markers in a mouse model of ALI.

IMPLICATIONS FOR PATIENT CARE: Quantitative ${ }^{64} \mathrm{Cu}$-LLP2A PET is a feasible and translatable approach for noninvasive tracking of lung inflammation and may be used toward a precision approach to ALI.

\section{REFERENCES}

1. Rubenfeld GD, Caldwell E, Peabody E, et al. Incidence and outcomes of acute lung injury. N Engl J Med. 2005;353:1685-1693.
2. Shaver CM, Bastarache JA. Clinical and biological heterogeneity in acute respiratory distress syndrome: direct versus indirect lung injury. Clin Chest Med. 2014;35:639-653.

3. Proudfoot AG, McAuley DF, Griffiths MJ, Hind M. Human models of acute lung injury. Dis Model Mech. 2011;4:145-153.

4. Johnson ER, Matthay MA. Acute lung injury: epidemiology, pathogenesis, and treatment. J Aerosol Med Pulm Drug Deliv. 2010;23:243-252.

5. Burnham EL, Janssen WJ, Riches DW, Moss M, Downey GP. The fibroproliferative response in acute respiratory distress syndrome: mechanisms and clinical significance. Eur Respir J. 2014;43:276-285.

6. Wu C, Evans CE, Dai Z, et al. Lipopolysaccharide-induced endotoxemia in corn oil-preloaded mice causes an extended course of lung injury and repair and pulmonary fibrosis: a translational mouse model of acute respiratory distress syndrome. PLoS One. 2017;12:e0174327.

7. Park PK, Cannon JW, Ye W, et al. Incidence, risk factors, and mortality associated with acute respiratory distress syndrome in combat casualty care. J Trauma Acute Care Surg. 2016;81(suppl):S150-S156.

8. Liu Y, Gunsten SP, Sultan DH, et al. PET-based imaging of chemokine receptor 2 in experimental and disease-related lung inflammation. Radiology. 2017;283: 758-768.

9. Han W, Zaynagetdinov R, Yull FE, et al. Molecular imaging of folate receptor beta-positive macrophages during acute lung inflammation. Am J Respir Cell Mol Biol. 2015;53:50-59.

10. Chen DL, Mintun MA, Schuster DP. Comparison of methods to quantitate ${ }^{18} \mathrm{~F}-$ FDG uptake with PET during experimental acute lung injury. $\mathrm{J}$ Nucl Med. 2004;45:1583-1590.

11. Braune A, Hofheinz F, Bluth T, et al. Comparison of static and dynamic ${ }^{18}$ F-FDG PET/CT for quantification of pulmonary inflammation in acute lung injury. J Nucl Med. 2019;60:1629-1634.

12. Tavakoli S, Downs K, Short JD, et al. Characterization of macrophage polarization states using combined measurement of 2-deoxyglucose and glutamine accumulation: implications for imaging of atherosclerosis. Arterioscler Thromb Vasc Biol. 2017;37:1840-1848.

13. Tavakoli S, Short JD, Downs K, et al. Differential regulation of macrophage glucose metabolism by macrophage colony-stimulating factor and granulocytemacrophage colony-stimulating factor: Implications for ${ }^{18} \mathrm{~F}$ FDG PET imaging of vessel wall inflammation. Radiology. 2017;283:87-97.

14. Tavakoli S, Zamora D, Ullevig S, Asmis R. Bioenergetic profiles diverge during macrophage polarization: implications for the interpretation of ${ }^{18} \mathrm{~F}$-FDG PET imaging of atherosclerosis. J Nucl Med. 2013;54:1661-1667.

15. Cao Q, Huang Q, Mohan C, Li C. Small-animal PET/CT imaging of local and systemic immune response using ${ }^{64} \mathrm{Cu}-\alpha \mathrm{CD} 11$ b. J Nucl Med. 2019;60:13171324 .

16. Kadioglu A, De Filippo K, Bangert M, et al. The integrins Mac-1 and alpha4beta1 perform crucial roles in neutrophil and $\mathrm{T}$ cell recruitment to lungs during Streptococcus pneumoniae infection. J Immunol. 2011;186:5907-5915.

17. Feng CG, Britton WJ, Palendira U, Groat NL, Briscoe H, Bean AG. Up-regulation of VCAM-1 and differential expansion of beta integrin-expressing T lymphocytes are associated with immunity to pulmonary Mycobacterium tuberculosis infection. J Immunol. 2000;164:4853-4860.

18. Walrath JR, Silver RF. The $\alpha 4 \beta 1$ integrin in localization of Mycobacterium tuberculosis-specific T helper type 1 cells to the human lung. Am J Respir Cell Mol Biol. 2011;45:24-30.

19. Koo GC, Shah K, Ding GJ, et al. A small molecule very late antigen-4 antagonist can inhibit ovalbumin-induced lung inflammation. Am J Respir Crit Care Med. 2003;167:1400-1409.

20. Bocchino V, Bertorelli G, D'Ippolito R, et al. The increased number of very late activation antigen-4-positive cells correlates with eosinophils and severity of disease in the induced sputum of asthmatic patients. J Allergy Clin Immunol. 2000;105:65-70.

21. Mishra A, Guo Y, Zhang L, et al. A critical role for $\mathrm{P} 2 \mathrm{X} 7$ receptor-induced VCAM-1 shedding and neutrophil infiltration during acute lung injury. J Immunol. 2016;197:2828-2837.

22. Li XC, Miyasaka M, Issekutz TB. Blood monocyte migration to acute lung inflammation involves both CD11/CD18 and very late activation antigen-4-dependent and independent pathways. J Immunol. 1998;161:62586264 .

23. Tsokos M, Fehlauer F. Post-mortem markers of sepsis: an immunohistochemical study using VLA-4 (CD49d/CD29) and ICAM-1 (CD54) for the detection of sepsis-induced lung injury. Int J Legal Med. 2001;114:291-294.

24. Choi J, Beaino W, Fecek RJ, et al. Combined VLA-4-targeted radionuclide therapy and immunotherapy in a mouse model of melanoma. J Nucl Med. 2018; 59:1843-1849. 
25. Beaino W, Nedrow JR, Anderson CJ. Evaluation of ${ }^{68} \mathrm{Ga}-$ and ${ }^{177}$ Lu-DOTAPEG4-LLP2A for VLA-4-targeted PET imaging and treatment of metastatic melanoma. Mol Pharm. 2015;12:1929-1938.

26. Beaino W, Anderson CJ. PET imaging of very late antigen-4 in melanoma: comparison of ${ }^{68} \mathrm{Ga}$ - and ${ }^{64} \mathrm{Cu}$-labeled NODAGA and CB-TE1A1P-LLP2A conjugates. J Nucl Med. 2014;55:1856-1863.

27. Soodgupta D, Zhou H, Beaino W, et al. Ex vivo and in vivo evaluation of overexpressed VLA-4 in multiple myeloma using LLP2A imaging agents. $\mathrm{J}$ Nucl Med. 2016;57:640-645.

28. Soodgupta D, Hurchla MA, Jiang M, et al. Very late antigen-4 ( $\alpha_{4} \beta_{1}$ integrin) targeted PET imaging of multiple myeloma. PLoS One. 2013;8:e55841.

29. Mattila JT, Beaino W, Maiello P, et al. Positron emission tomography imaging of macaques with tuberculosis identifies temporal changes in granuloma glucose metabolism and integrin $\alpha 4 \beta 1$-expressing immune cells. J Immunol. 2017;199:806-815.

30. Cross LJ, Matthay MA. Biomarkers in acute lung injury: insights into the pathogenesis of acute lung injury. Crit Care Clin. 2011;27:355-377.
31. Trotta T, Di Gioia S, Piro D, et al. Effect of acute lung injury on VLA-4 and CXCR4 expression in resident and circulating hematopoietic stem/progenitor cells. Respiration. 2013;85:252-264.

32. Burns JA, Issekutz TB, Yagita H, Issekutz AC. The $\alpha_{4} \beta_{1}$ (very late antigen (VLA)-4, CD49d/CD29) and $\alpha_{5} \beta_{1}$ (VLA-5, CD49e/CD29) integrins mediate $\beta_{2}$ (CD11/CD18) integrin-independent neutrophil recruitment to endotoxin-induced lung inflammation. J Immunol. 2001;166:4644-4649.

33. Peng L, Liu R, Marik J, Wang X, Takada Y, Lam KS. Combinatorial chemistry identifies high-affinity peptidomimetics against $\alpha_{4} \beta_{1}$ integrin for in vivo tumor imaging. Nat Chem Biol. 2006;2:381-389.

34. Kenyon NJ, Liu R, O'Roark EM, Huang W, Peng L, Lam KS. An $\alpha 4 \beta 1$ integrin antagonist decreases airway inflammation in ovalbumin-exposed mice. Eur $J$ Pharmacol. 2009;603:138-146.

35. de Souza Xavier Costa N, Ribeiro G Junior, Dos Santos Alemany AA, et al. Early and late pulmonary effects of nebulized LPS in mice: an acute lung injury model. PLoS One. 2017;12:e185474. 